\title{
Interactive Accessible Notifications for Emergency Notification Systems
}

\author{
Alessio Malizia ${ }^{1}$, Teresa Onorati ${ }^{1}$, Andrea Bellucci $^{2}$, Paloma Diaz $^{1}$, and Ignacio Aedo ${ }^{1}$ \\ ${ }^{1}$ Universidad Carlos III de Madrid, Computer Science Dept., Avenida de la Universidad 30, \\ 28911, Leganés, Madrid, Spain \\ \{amalizia, tonorati,pdp\}@inf.uc3m.es, aedo@ia.uc3m.es \\ ${ }^{2}$ Sapienza Universita' di Roma, Computer Science Dept., Via Salaria 113, \\ 00198, Roma, Italy \\ bellucciedi.uniromal.it
}

\begin{abstract}
Notifications are critical when an emergency scenario is going to happen (e.g. a hurricane approaching); so the ability to transmit notifications to different kind of users is a crucial feature for Emergency Management Systems. In this work an ontology was developed by investigating different sources: accessibility guidelines, emergency response systems, communication devices and technologies, taking into account the different abilities of people to react to different alarms (e.g. mobile phone vibration as an alarm for deaf blind people). The knowledge codified in the proposed ontology could be used to enhance and promote the use of non-conventional interfaces for notifying emergency messages thus providing accessibility under different conditions and for different kind of users.
\end{abstract}

Keywords: Accessibility, Eye-tracking, Universal Design, Non-conventional interfaces, Emergency Notification Systems (ENS).

\section{Introduction}

When a disaster occurs it is very important that emergency response information systems share a common knowledge to support their disaster management alerting functions and notifications. Notifications are critical in an emergency scenario (e.g. a typhoon approaching); so it is crucial, for emergency systems, to be able to transmit notifications to all kinds of recipients. It is important to take into account that recipients are not only information systems but also the people who will be affected by the emergency as well as those in charge of the response. Moreover, in this last group there are many kinds of organizations and communities that use different devices and systems and have also different levels of expertise in emergency management. In this work we propose an approach that addresses the information needs for adapting emergency notification messages and contents to be accessible and interactive for different kind of users. An ontology based approach was developed by investigating different sources: accessibility guidelines, emergency notification systems, communication devices and technologies; and taking into account the different abilities of people to 
react to different alarms, thus using non-conventional interaction techniques for helping vulnerable collectives.

Our approach has been developed by investigating different communities (blinds, elderly, disabled, etc.), for codifying a knowledge base of different abilities to react to different alarms (e.g. during a fire alarm the system can send a video showing emergency exits to deaf people that could not follow sound alerts). Moreover we developed an ontology-driven approach that automatically adapts emergency information to be managed by non-conventional interaction techniques.

We think that non-conventional interaction approaches can be of great help in case of notifications to vulnerable groups. Let's imagine deaf people that can receive an emergency notification through the vibration of a mobile devices or PDA (Personal Digital Assistant). This notification could open up a multimedia message including text describing the emergency and for example a map indicating a route to follow for evacuation; the user can then interact with the device by looking at different part of the screen and zooming automatically on text or images (map portions) of interest. In fact the built-in camera that mobile device or personal digital assistant (PDA) use to include can be exploited to detect the eyes movement and, automatically, zoom text or map portions. This will help vulnerable collective, like people with physical impairments or elderly that have problems in pressing small buttons, or even people in a stress or panic situation (we are all disabled in some way during an emergency).

We focused in aspects that have not traditionally been central to human-computer interaction; these aspects are now emerging as important factors in the design of critical and emergency information systems taking into account vulnerable and special needs communities of users, like: the user experience related to emergency and security, adaptive user interfaces, non-conventional interaction, enabling tools for social responsiveness and reaction, accessible alerting systems for communities with special needs (disabled people, elders, ...), and, finally, universal design.

In this paper we will introduce accessibility in emergency notifications system, and then we will describe the ontology based approach used for adapting the notifications depending on the users' abilities, the devices they can access and the kind of alert notification. We will conclude the paper illustrating a scenario where such approach, together with the use of non-conventional interaction can be of great help for sending adaptable notification to users.

\section{Accessibility in Emergency Notification Systems}

The problem of the accessibility, no longer consists of simply adopting measures that compensate the disadvantages or that surpass the functional limitations of people with disabilities. In fact, new approaches deal with separating accessibility from disability and extending it to all population in favor of the new paradigm of "Universal Design" or "Design for All". Design for all, comes from a simple idea: to make it possible that products and services can be used or acceded by all. Although the concept of "Design for all" represents an important part of accessibility, it cannot replace the concept of accessibility since it is also about the adaptation to different users' characteristics. Adapting the environment to be accessible, not only is good for people with disabilities 
but also for those without disabilities, this is remarkable if we think about urban adaptation in big cities.

When managing emergencies, accessibility is a crucial issue to save lives and protect from damages vulnerable collectives. In particular we focused on a specific feature of Emergency Management Systems (EMS) which is alert notification. Within EMS there exist a subclass of Emergency Notification Systems (ENS) which in some cases is a separate system and in others is a module of an EMS. They are generally effective for notifying emergency alerts to registered people by using different media, such as: sms, mms, e-mail or Internet when available.

ENS are intended to deliver information to as many devices as possible, in order to reach people using different kinds of hardware. However, to our knowledge all the existing emergency management systems do not include or include only partially accessibility aspects to generate an effective message format according to people's profiles and preferences, as surveyed in [1].

Nevertheless we can conclude that not only the emergency notifications depend on people's abilities and the devices characteristics, but also on the kind of emergency that could inhibit the device capabilities or change the user's characteristics (let's imagine people during a fire emergency where their vision is inhibited by the smoke).

\section{The SEMA4A Ontology}

Creating ontologies is not a simple task. Furthermore, once an ontology has been created, it must be validated by a scientific community or by related fields experts.

In [2] Berners-Lee reports on the relevancy of interoperability in emergency and disaster situations. He states that disaster response is strictly related to preparedness and highlights the importance of the development of disaster management ontologies. As an example, the availability of relevant data in a machine readable format (XML, RDF, OWL, etc) could be helpful for those in the ground, in order to better decide the kind of actions to attempt. Efforts in exploiting ontology for crisis management include the OpenKnowledge system, which supports and enhances the sharing and effective use of information and services among different actors [3]. Previous work also focused on blogs and the collaborative tagging approach [4]. The present work go further, proposing an ontological approach to enable the use of non-conventional interfaces for notifying emergency messages, enhancing accessibility. Recent contributions on developing ontologies for crisis knowledge representation can be found in [5] and [6]. In [5] an adaptive ontology for assisting in coordinating relief efforts in different crisis situations is presented, while in [6] the authors discuss how ontologies can be used, in conjunction with standard messaging formats, to enable intelligent decision support mechanisms.

We give, here, a brief description of the main classes and concepts included in the SEMA4A (Simple EMergency Alerts fo[u]r All) ontology to give an overall idea of the specific knowledge codified in it. The main classes are: AccessOnto (including concepts on disabilities and accessibility guidelines), WAFa (including structural definitions for formatting content on the web, to which usually guidelines are applied), and EMEDIA (including concepts and relations on emergency and communication devices and technologies). 
The Web Authoring for Accessibility (WAfA) is an existing ontology also known as Travel Ontology because it is based on the analogy of web navigation with tourists' trips. This ontology represents concepts and relations necessary to automatically model the structural organization and navigation of web pages [7] to users' profiles. This ontology has been evaluated with real users, contains information on how to model content for being accessible, and it is codified using the Web Ontology Language (OWL) ${ }^{1}$ for its knowledge representation capabilities and for being one of the most used to represent web ontologies; we extended our ontology including WAfA concepts, defining a class called WAfA that contains concepts and relations needed to model organization, structure and navigation of sites.

AccessOnto in an ontology in the form of an accessibility requirements repository from which it is possible to extract requirements using an accessibility knowledge base (AKB) built on user's characteristics [8]. It includes guidelines from Web Accessibility Initiative, Sun Micro Systems, IBM, Microsoft, and Apple guidelines. In our ontology we created a class called AccessOnto that contains information related to Web accessibility guidelines, users' profiles and actions that users can perform. We created this class translating information from XML (AccessOnto is codified in XML) to OWL; after this phase, we established relations that linked concepts contained in WAfA and in AccessOnto sections, as we will show in the use case section.

EMEDIA (Emergency and MEDIA technologies) is the portion of the SEMA4A ontology that provides concepts and relations about emergency and media technologies. We developed it trough a semiautomatic procedure with two phases: the first phase was performed to extract new concepts and relations from WordNet [9] (concerning emergency and media technologies); the second to integrate new information within the existing ontology (adding relations with the others portions). We applied this technique to develop and expand part of our ontology related to the emergencies and how they can affect technologies accessible to the users [1].

Summarizing, SEMA4A counts on three basic classes: WAfA, AccessOnto and EMEDIA; it includes information related to concepts and relations needed to model organization, structure and navigation of information contents; accessibility guidelines, user's profiles and actions that users can perform; as well as information related to emergencies, notifications and devices. These main classes are linked with relations existing within their subclasses. In the following section we provide a use case that depicts the more common relations that exist in our ontology and how to use it to adapt notifications for accessibility.

\section{Accessible Alerts through Non-conventional Interfaces}

In this section we present an example of using non-conventional interaction for notifications within vulnerable groups. Let us image that Maria is an elderly person with limited motor abilities, who lives in 38255 Blacow Rd, Fremont, CA 94536, United States. Maria's wheelchair is equipped with a mobile device (e.g. PDA - Personal Digital Assistant) and she has been subscribed to an ENS (Emergency Notifications System) by her relatives or assistants. In her profile, the PDA is setup as the preferred

\footnotetext{
${ }^{1}$ http://www.w3.org/TR/owl-guide/
} 
device to receive alerts. Her PDA includes various features: internet connection, vibration, short and multimedia message service and eye tracking. By eye tracking we mean software that detects the eyes movements using a camera. Information about eyes movements could be applied to detect user's focus of attention. In this way, the eye tracking can help interacting with the PDA: for example, the usage of mouse or keyboard with hands could be replaced by detecting eyes movements on the display.

The eye tracking could allow navigating many different kinds of information that she could receive: texts, figures and videos. For example, if the message content is a figure representing a map, she can zoom or scroll it by using her eyes. Moreover, she can take a virtual walk-through in a map just using her eyes: the image in the display changes to show the next step of the virtual walk-through depending on where she is looking at; this could help in finding the right turns to take in order to follow a suggested route. Finally, if emergency sites (like hospitals or civil protection centres) are visualized on the map (as points with special icons), the user can see how to go there during her virtual walk-through.

Consider the case in which an earthquake strikes the San Francisco Bay area, where Maria lives. In this example, Maria is a person on a wheelchair with limited motor capacities, but the same scenario holds for other kinds of abilities (e.g. elderly with arthritis may have difficulty in using their hands). She is subscribed to an ENS that sends an alert to her: it communicates that an earthquake has damaged various areas of the city and an emergency plan is available. The PDA vibrates to notify that a new message has arrived; the PDA shows information about the earthquake and the map with safe areas, hospitals and civil protection centres (by using the content of the received message). In order to clarify the relations among different concepts within the SEMA4A ontology, we exploit a graphical formalism (as shown in Figures 1-4). Each box represents a class of the ontology, with its subclasses. The edges represent relations between classes. As an example, in Figure 1, Coordination_difficulty is a subclass of Motor (a subclass of Impairment). Defined relations are mayHaveDifficulty with mouse and keyboard (subclasses of conventional in Interaction); or mayUse with eye_tracking, speech_input and speech output (subclasses of non-conventional in Interaction), sms and mms (subclasses of transmission in Media). Moreover, sms can communicate with Text (subclass of Contents) and mms can communicate with all subclasses of Contents. In the graphical representation an arrow pointing to a class (external border) implies that the relation holds for the whole class, while an arrow pointing directly to a concept (an element) means that the relation is restricted to the specific instance of the concept.

The ENS could use SEMA4A to access information about the profile of the users (kinds of abilities) and the specific emergency. The first information that can be derived from the ontology is about the disability of the person. The coordination difficulty is a motor impairment where people have difficulty with all devices that require an interaction with hands (as shown in Figure 1). For this reason, they cannot use a mouse or a keyboard, but they can interact with eyes using the eye-tracking. Furthemore, from the class Motor of the ontology we obtain that a person with coordination difficulty could access information contained in sounds, videos, figures, texts, short and multimedia message service and device vibrations. 


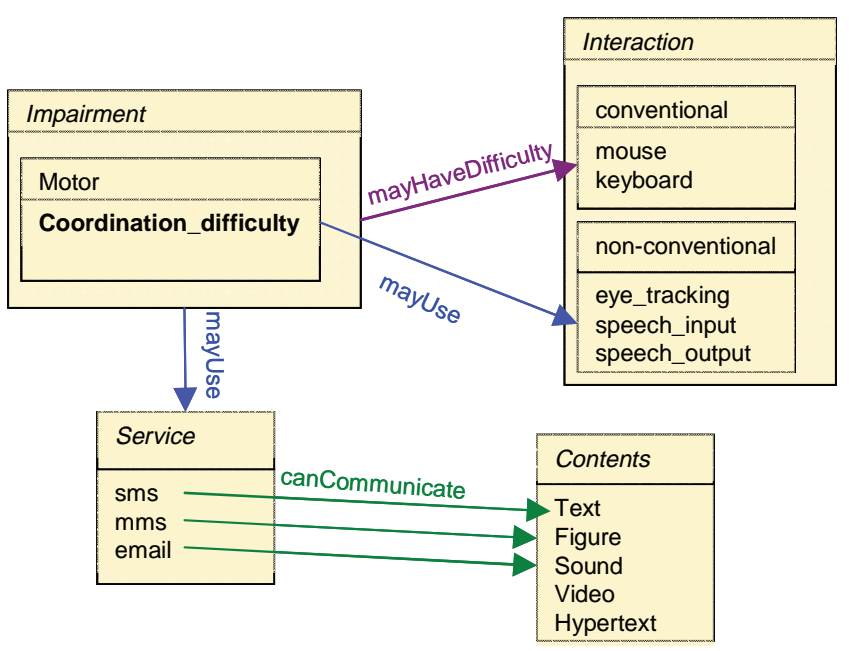

Fig. 1. The definition of Coordination_difficulty

Successively, the ENS obtains from the ontology that the PDA, the device used by the involved person, is a media device, also known as personal digital assistant or personal organizer, with various features: it can communicate with figures, texts, vibration signals and recorded sounds (see Figure 2).

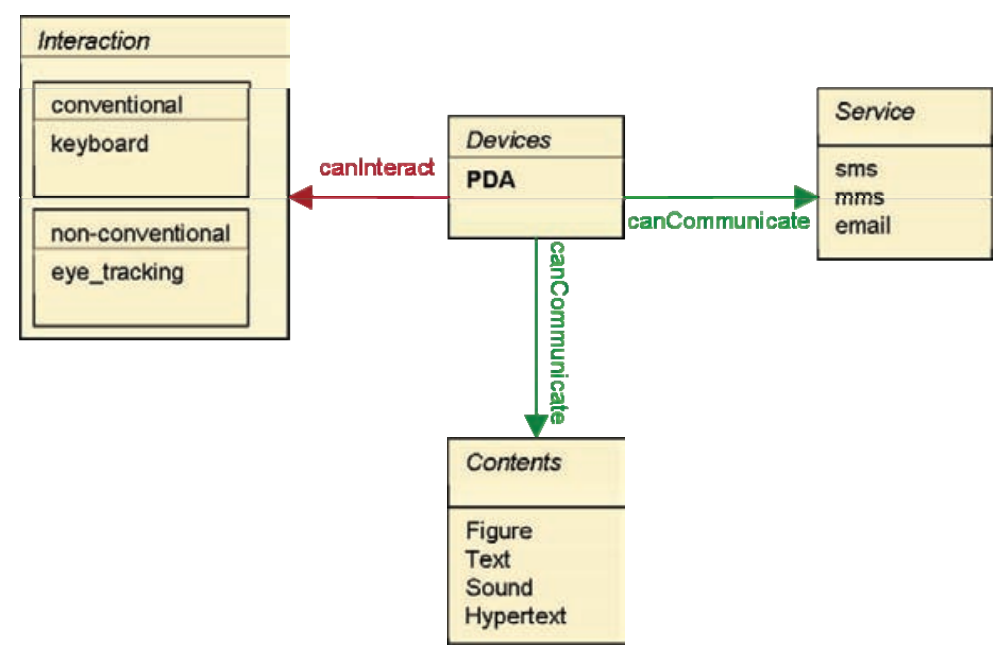

Fig. 2. PDA communication features. PDA can communicate with email, sms, mms and it can interact with keyboard (conventional interaction) and eye_tracking (non-conventional interaction).

Moreover, information about the emergency are also included in SEMA4A: in our case an earthquake (see Figure 3). An earthquake is a particular emergency that can be notified through media, like a television, a radio, an internet message, a phone or mobile phone. The available information can also be consulted with an eye tracking as a non-conventional way to interact with the video camera and the appropriate software. In our specific case, the person involved in the emergency cannot use her hands and for this reason she interacts with her eyes: employing the eye tracking, eyes movements can substitute the mouse and the keyboard. 


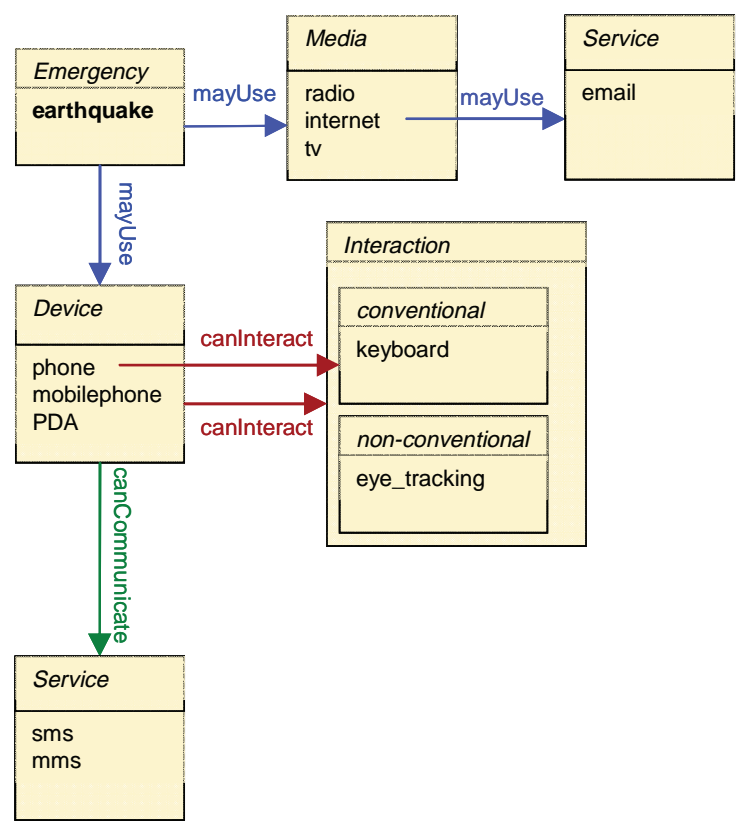

Fig. 3. The definition of the earthquake with media and devices used to alert

At this stage the ENS can retrieve information from SEMA4A about the alert to transmit. The person, in our case, is using a PDA connected to internet: the ENS can send the alert as an internet message (e.g. an e-mail). From the ontology, we can obtain the kind of information that the message can send: texts, figures, videos or sounds (see Figure 4).

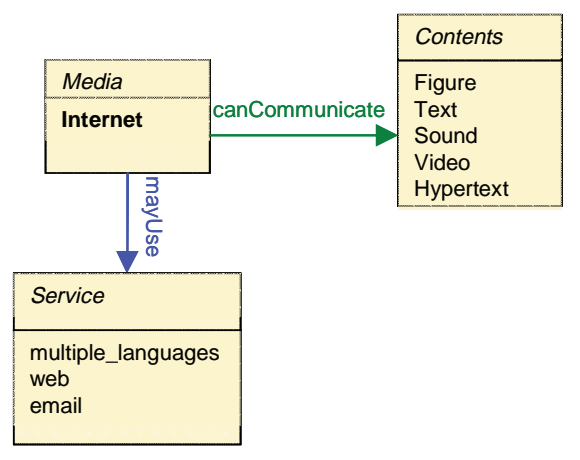

Fig. 4. Internet characterization

In this example, the message sent by the ENS contains an image with the map of the emergency plan and textual information. The map can be a simple image, an interactive image or a image based service, like the one available at StreetView ${ }^{2}$ of GoogleMaps. For example, an interactive map could allow a virtual walk-through between two points: in our case, Maria can visualize the route between her position (e.g. her house) and an emergency site (e.g. the Prince of Peace Lutheran Church, 38451 Fremont Blvd, Fremont, CA 94536 where the civil protection has established a first-aid center). With the eyes movement she can navigate virtually along this route, turning left or right (by looking left or right) and going back or on (by looking down

\footnotetext{
${ }^{2}$ maps.google.com/streetview
} 
or up). Another possible functionality can be the direct interaction with icons of emergency sites: she could decide to call a hospital indicated on the map just with a special sequence of eyes movements (e.g. a wink).

The disability chosen in this example does not have a set of guidelines that could guarantee the accessibility of information. To ensure that the user can access to proposed information, the ENS has to adapt the message, depending on the emergency and the device she is using. In case of a person with coordination difficulty, the used device depends on the kind of difficulty. If she has difficulty using hands, as in our example, she needs a non-conventional interaction to access her device that does not involve hands. The eye tracking is an example of non-conventional interaction that substitutes the mouse and the keyboard with the eyes movements [10,11]. A similar scenario can involve an elderly person with arthritis and limited motor capacities. Moreover, here we have considered a PDA, but the eye tracking can be used with any kind of devices that have a camera and appropriate software.

In Figure 5 we can see an example of an eye tracker developed by us (using the OpenCV ${ }^{3}$ libraries and Google Streetview ${ }^{4}$ APIs) to synchronize the eye movements with the walk-through presented to the user. The mobile device (the Ultra-Mobile PC in Figure 6), equipped with a camera and the eye-tracking application, allows the user to check which street she has to take in order to follow the evacuation plan, by providing a view at street level. A wheelchair could be easily equipped with such device, for guiding the user through the streets of a city and interacting without hands by employing only the head and eye movements.

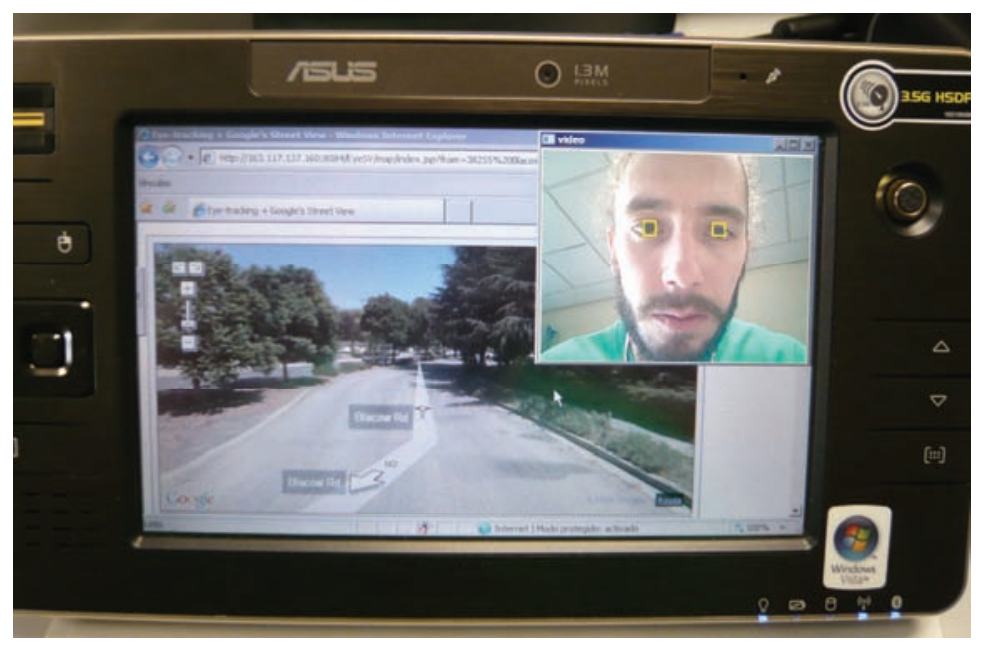

Fig. 5. The eye-tracker together with Google streetview running on an Ultra-Mobile PC equipped with wifi connection

\section{Future Evaluation}

The goal of this work is to determine whether the adaptation to users' abilities can improve the notification of emergencies. We are planning an evaluation to

\footnotetext{
${ }^{3}$ OpenCV are a set of open-source libraries for developing Computer Vision and Pattern Recognition algorithms in C; available at sourceforge.net/projects/opencvlibrary

${ }^{4}$ Google Streetview Application Programming Interfaces are part of Google maps API; available at google.es/apis/maps
} 
demonstrate that interactive accessible notifications can help people to reach emergency point quickly.

We will consider just one independent variable: the type of notifications. The classic one is a standard message about the emergency, sent via radio or via web services. Notifications we proposed here are adapted to the user's profile and the occurred emergency. The location of the evaluation will be the central square of Leganés, the site of University campus. We will not locate the evaluation in a building of the University campus because we may have problems with devices that need GPS.

We are planning to simulate an emergency fire and to ask to involved students to go from the square to the nearest hospital. They will perform this task under two different conditions: receiving notifications from a classic system and receiving interactive accessible notifications. In the first case, they will receive a message with general information about the emergency and they have to find additional specification, like the nearest emergency point, calling to an emergency number. In the second case, received information depends on the used devices and the particular emergency. For example, if one of students has a PDA, she can receive an email with a map and all emergency points visualized.

In order to compare the performance of the students in these two different situations, we will select the arrival time to the hospital and the difficulties met along the route as dependent variables and metrics. At the end of the evaluation, we will analyze obtained results looking at these variables. The objective is to confirm the initial hypothesis: accessible notifications permit to reduce the arrival time to an emergency point in case of people with limited abilities.

\section{Conclusion and Future Works}

In this paper we presented an approach for managing accessible notifications through non-conventional interfaces for emergency management systems. We designed and developed an ontology (SEMA4A) made of three integrated parts in order to automatically adapt emergency notifications to users' abilities, kinds of devices they could use, and the type of emergency they are involved in. The information codified in the ontology could be used to adapt notifications to different devices accessible through different interaction paradigms, depending on the user's abilities. In the example shown in this paper we used information to adapt notification for a user with motor impairment, presenting a scenario in which the user receives an evacuation plan that could be accessed by a PDA and an eye tracker to compensate her disabilities. The use of non-conventional interfaces, like the eyes movements for navigating Google Street images and directions, is an emerging field raising interest among researchers and companies, like Microsoft that has, recently, released a library called Touchless 5 which allow users to interact with recognized object through a simple webcam.

Finally, we intend to extend the models and classes of non-conventional interfaces and paradigms in our ontology building relations with classes of users' abilities and emergency characteristics to automatically adapt notifications for a wider set of users and innovative devices.

\footnotetext{
${ }^{5}$ http://www.codeplex.com/touchless
} 
Acknowledgments. This work is funded by the project UIA4SIGE (TSI2007-03394) supported by the Ministry of Education and by an agreement with the DGPCE of the Ministry of Interior.

\section{References}

1. Malizia, A., Astorga, F., Onorati, T., Diaz, P., Aedo, I.: Emergency Alerts for All: an Ontology based Approach to Improve Accessibility in Emergency Alerting Systems. In: ISCRAM 5th International Conference Information Systems for Crisis Response and Management, vol. 1, pp. 197-207 (2008)

2. Berners-Lee, T.: Disaster Management Ontologies (2007), http://lists.w3.org/ Archives/Public/semantic-web/2007Apr/0094.html

3. Vaccari, L., Marchese, M., Giunchiglia, F., McNeill, F., Potter, S., Tate, A.: OpenKnowledge Deliverable 6.5: Emergency response in an open information systems environment (2006)

4. Ziesche, S.: Social-networking web systems: Opportunities for humanitarian information management. Journal of Humanitarian Assistance (2007)

5. Segev, A.: Adaptive Ontology Use for Crisis Knowledge Representation. In: ISCRAM 5th International Conference Information Systems for Crisis Response and Management, vol. 1, pp. 285-293 (2008)

6. Lam, H.P., Steel, J., Iannella, R.: Using Ontologies for Decision Support in Resource Messaging. In: ISCRAM 5th International Conference Information Systems for Crisis Response and Management, vol. 1, pp. 276-284 (2008)

7. Yesilada, Y.: Annotation and transformation of Web pages to improve mobility for visually impaired users. Ph.D Thesis. School of Computer Science at the University of Manchester, UK (2005)

8. Masuwa-Morgan, K.R., Burrell, P.: Justification of the need for an ontology for accessibility requirements (Theoretic framework). Interacting with Computers 16(3), 523-555 (2004)

9. Miller, G.A., Beckwith, R., Felbaum, C., Gross, D., Miller, K.: Introduction to WordNet: An On-line Lexical Database. International Journal of Lexicography 3(4), 235-244 (1990)

10. Park, K.S., Lim, C.J.: A Simple Vision-Based Head Tracking Method for Eye Controlled Human/Computer Interface. Int. J. Human-Computer Studies 54, 319-332 (2001)

11. Cleveland, N.R.: Eyegaze Human-Computer Interface for People with Disabilities. In: 1st Conference on Automation Technology and Human Performance (1997),

http://www.eyegaze.com/doc/cathuniv.htm 